\title{
Motivos para evasão, vivências acadêmicas e adaptabilidade de carreira em universitários
}

\author{
Rodolfo Augusto Matteo Ambiel \\ Acácia Aparecida Angeli dos Santos \\ Universidade São Francisco, SP, Brasil \\ Simone Nenê Portela Dalbosco \\ Faculdade Meridional, RS, Brasil
}

\begin{abstract}
Resumo
O estudo tem como objetivo avaliar se a vivência acadêmica e a adaptabilidade de carreira estão associadas aos motivos para evasão no ensino superior, conforme o curso e a etapa de formação. A amostra foi de 153 universitários de uma instituição particular localizada em uma cidade no interior de São Paulo e foram utilizados os instrumentos Questionário de Vivência AcadêmicaReduzido (QVA-r), Escala de Adaptabilidade de Carreira (CAAS) e Escala de Motivos para Evasão do Ensino Superior (M-ES). Os dados foram analisados através de estatísticas descritivas, correlação de Pearson e regressão linear múltipla. Os resultados mostraram que o modelo incluindo os fatores do QVA-r e da CAAS simultaneamente é o mais adequado na predição dos fatores da M-ES de motivos relacionados à Carreira, ao Desempenho Acadêmico, ao Interpessoal e à Autonomia, explicando entre 14,3\% e 19\% da variância. Os resultados são discutidos à luz da teoria e de suas implicações práticas.
\end{abstract}

Palavras-chave: Adaptação; Desenvolvimento de carreira; Ensino superior.

\section{Reasons for avoidance, academic experiences and career adaptability in university}

\begin{abstract}
The study aims to evaluate if the academic experience and career adaptability are associated with reasons for dropout in the higher education, as graduation and the formation stage. The sample consisted of 153 students from a private institution located in a town in São Paulo and the instruments used were a socioeconomic questionnaire, Reduced-Academic Experience Questionnaire (QVA-r), Career Adaptability Scale (CAAS) and Range of Reasons for Avoidance of Higher Education (M-ES). Data were analyzed using descriptive statistics, Pearson correlation and multiple linear regression. The results showed that the model including QVA-r and the CAAS factors simultaneously is the most adequate for predicting the M-ES Factors reasons related to career, the Academic Performance, the Interpersonal and autonomy, explaining between $14.3 \%$ and $19 \%$ of the variance. The results are discussed in light of theory and its practical implications.
\end{abstract}

Keywords: Adaptation; Career development; Higher education.

\section{Razones de la evasión, experiencias académicas y adaptabilidad carrera en la universidad \\ Resumen}

El estudio avalúa sí la vivencia académica y la adaptabilidad de carrera están asociadas a los motivos para la evasión en la enseñanza superior privada de una muestra de 153 universitarios. Los instrumentos de recogida de los datos fueron, un Cuestionario socioeconómico, Cuestionario de la Vivencia Académica-Reducido (QVA-r), Escala de Adaptabilidad de Carrera (CAAS) y Escala de Motivos para la Evasión de la Enseñanza Superior (M-ES). A través de la estadística descriptiva, de correlación de Pearson y regresión lineal múltiple, concluyese que el modelo, incluyendo QVA-r y la CAAS juntos, es lo más adecuado para predecir la M-ES, factores de razones relacionadas con la Carrera, el Rendimiento Académico, Interpersonal y la Autonomía, explicando entre el $14,3 \%$ y el $19 \%$ la varianza.

Palabras clave: Adaptabilidad; Desarrollo profesional; Enseñanza superior. 


\section{Introdução}

O crescimento do Ensino Superior no Brasil ocorreu pelo incentivo de Leis Federais à iniciativa privada, gerando o acréscimo de vagas e facilitando que um número maior de estudantes chegasse ao ensino superior (Brasil, 2005, 2007). Assim como em outros países, além do lugar social que lhe é conferido, ao entrar no mundo universitário, as demandas decorrentes da nova realidade exigem que o jovem enfrente os inúmeros desafios do contexto acadêmico (Araújo et al., 2015). Também nesse período o estudante se prepara para enfrentar os desafios relacionados à construção de sua carreira futura, desenvolvendo habilidades técnicas e pessoais para passar pelas transições entre os estudos e o trabalho (Monteiro \& Almeida, 2015). Contudo, a conclusão do curso é uma prerrogativa que nem todos os estudantes conseguem atingir, o que pode se dever a diversos motivos (Cabrera, Bethencourt, Pérez, \& Afonso, 2006; Iñiguez, Saso \& Errazu, 2016). Dessa forma, no presente trabalho objetivou-se verificar o quanto variáveis de adaptação acadêmica e à carreira são capazes de predizer motivos para evasão do ensino superior.

Partindo do pressuposto que a entrada na universidade representa uma conquista pessoal e social, o aluno necessita adequar-se às novas circunstâncias. Este processo chamado de adaptação acadêmica é essencial para lidar com as situações novas e superar as adversidades provindas do começo da vida universitária. A literatura sobre o tema tem definido a adaptação acadêmica como um processo multifacetado, que exige do aluno o ajustamento à instituição de ensino, suas normas e valores. Indica também que a busca dos recursos intrapessoal, interpessoal e familiar será necessária para a própria adaptação ao curso, desde os anos iniciais até a formatura. Exige, ainda, a assunção de um papel responsável e o envolvimento proativo nas resoluções quanto à carreira para alcançar o que o aluno almejou com o curso escolhido (Almeida, Ferreira \& Soares, 2003; Astin, 1993; Chickering \& Reisser, 1993; Tinto, 1993).

A necessidade de implementar estudos com foco na adaptação acadêmica foi contemplada através da pesquisa de Almeida, Ferreira e Soares (1999). Os autores construíram e validaram o QVA-r (Questionário de Vivências Acadêmicas na versão reduzida) com uma amostra de 1.273 alunos universitários em Portugal. O estudo encontrou cinco dimensões da adaptação acadêmica, ou seja, pessoal, interpessoal, institucional, carreira e estudo. Respectivamente, tais dimensões dizem respeito à adaptação quanto à demandas emocionais do ensino superior; às relações estabelecidas com colegas, professores e demais membros da comunidade acadêmica; às regras, trâmites e ambiente da instituição como um todo; às expectativas e comportamentos efetivos em relação à carreira futura; e às rotinas e exigências de estudo do ensino superior (Almeida et al., 1999).

No Brasil, a validação do instrumento acima referido foi realizada por Granado, Santos, Almeida, Soares e Guisande (2005) com uma amostra de 626 alunos de duas universidades, uma pública e outra privada. A análise fatorial corroborou os cinco fatores da escala original portuguesa, explicando $40,4 \%$ da variância total. Os autores salientaram que houve a necessidade da exclusão de itens na adaptação brasileira, cuja configuração final ficou constituída por 55 itens. Desde então, outros estudos foram realizados no Brasil, atestando a validade do instrumento (Igue, Bariani \& Milanesi, 2008; Noronha, Martins, Gurgel, \& Ambiel, 2009; Oliveira \& Morais, 2015; Silva, Coelho, \& Teixeira, 2013; Teixeira, Castro, \& Piccolo, 2007).

Um destes estudos investigou a associação entre vivência acadêmica e desenvolvimento de carreira, bem como possíveis diferenças relativas à sexo, idade e etapa do curso em 208 universitários, de universidades particulares de São Paulo e Minas Gerais. Como resultados, os autores destacaram que as vivências acadêmicas são essenciais, não só para a adaptação do aluno à Universidade, mas para o desenvolvimento e investimento na carreira. Efetivamente, na literatura, a adaptação acadêmica e a adaptabilidade de carreira são construtos positivamente relacionados, e considerados fundamentais para que o aluno chegue ao final do curso e inicie sua carreira profissional (Sousa, Bardagi, \& Nunes, 2013).

O conceito de adaptabilidade de carreira foi proposto por Savickas (1997), e está relacionado às responsabilidades específicas das tarefas e à capacidade flexível para efetivar as mudanças necessárias relacionadas à sua carreira. Mais tarde, Savickas (2005) definiu adaptabilidade de carreira como um construto psicossocial que denota a prontidão de uma pessoa para lidar com tarefas de desenvolvimento de carreira, transições ocupacionais e traumas pessoais.

Segundo Savickas e Porfeli (2012), a adaptabilidade de carreira é composta por quatro dimensões, a saber, a Consideração quanto ao desenvolvimento de competências para planejar, o futuro da carreira escolhida, com o estabelecimento de metas e objetivos pessoais e profissionais; o Controle para construir com responsabilidade a própria carreira e ser capaz de tomar decisões; a dimensão da Curiosidade que está 
baseada na busca de novos aprendizados, envolvendo autoconhecimento e o conhecimento sobre o mundo organizacional; e a quarta dimensão é a da Confiança que trata sobre a crença do sujeito em sua própria competência para atingir os objetivos, mesmo com os desafios e obstáculos enfrentados com a carreira escolhida. O instrumento que permite realizar a avaliação da adaptabilidade de carreira é denominado de Career Adapt-Abilities Scale - CAAS (Escala de Adaptabilidade de Carreira - EAC; Savickas \& Porfeli, 2012). Nesta pesquisa será utilizada a versão brasileira de Teixeira, Bardagi, Lassance, Magalhães e Duarte (2012). Na literatura, são encontradas evidências favoráveis sobre o uso do instrumento com estudantes universitários, relacionando-o com satisfação acadêmica (Duffy, Douglass, \& Autin, 2015) e expectativas positivas de transição da universidade para o mercado de trabalho (Monteiro \& Almeida, 2015).

Se a adaptação acadêmica é o processo que ocorre em todas as fases da graduação e a adaptabilidade de carreira está relacionada com a transição da universidade ao mercado de trabalho, um obstáculo que pode se interpor a esta trajetória é a evasão, ou seja, a saída do curso antes de sua conclusão. Existem múltiplos motivos para a desistência de um curso, tais como a escolha equivocada, a desadaptação ao curso, falta de integração com professores e colegas, entre outros (Bardagi \& Hutz, 2005, 2014). Tinto (1975, 1993, 2007), um dos principais pesquisadores do fenômeno da evasão, privilegiou o estudo do tema a partir dos aspectos contextuais em que as questões econômicas e também as questões estruturais da universidade estão envolvidas na decisão do aluno pela evasão. Segundo o autor, aspectos interpessoais, como a falta de apoio familiar e integração social, com professores, colegas e com a universidade poderiam interferir também na decisão para uma evasão.

No Brasil, o fenômeno da evasão é uma preocupação relativamente recente de todas as instituições de ensino, tanto públicas quanto privadas. Em 1995, houve a criação da Comissão Especial de Estudos sobre Evasão por meio da portaria MEC/SESU (Brasil, 1996), cujo objetivo era o de desenvolver pesquisas sobre o desempenho das Instituições Federais de Ensino Superior (Bardagi \& Hutz, 2005).

O MEC/SESU (Brasil, 1996), definiu a evasão como o abandono do curso antes de sua conclusão, que ocorre por motivações individuais, dificuldades financeiras ou combinação das estruturas curriculares e métodos pedagógicos que falham no processo da aprendizagem. Nesse documento, o MEC sinaliza sobre três tipos de evasão, quais sejam, o abandono, que ocorre quando o aluno deixa de matricular-se para o próximo período; a desistência do curso, que é caracterizada por pedido formal de desligamento; e transferência ou reopção, quando ocorre uma mudança de curso. Outra característica importante do fenômeno é que pode ocorrer em dois níveis, que são a evasão da instituição, quando o estudante desliga-se da instituição na qual está matriculado podendo transferirse para outra, e a evasão do sistema, quanto o estudante abandona de forma definitiva ou temporária o ensino superior.

Silva Filho, Motejunas, Hipólito e Lobo (2007) definem dois aspectos sobre a mensuração da evasão. A evasão anual verifica a diferença entre alunos matriculados de um ano para o outro, e a evasão total, que se dá ao comparar o número de alunos matriculados com o número final de alunos concluintes do curso. $\mathrm{Na}$ pesquisa, os autores, registraram que no período compreendido entre 2000 e 2005 , no conjunto formado por todas as Instituições de Ensino Superior (IES) do Brasil, a evasão total média foi de $22 \%$ e atingiu $12 \%$ nas públicas e $26 \%$ nas particulares. Os autores também revelaram que são poucas as instituições que possuem um programa institucional regular de combate à evasão, com planejamento de ações, acompanhamento de resultados e coleta de experiências bem-sucedidas (Silva Filho et al., 2007).

Nas IES privadas, a evasão representa uma redução de receita, uma vez que os evadidos param de contribuir com as mensalidades, podendo chegar a inviabilizar alguns cursos de graduação. No âmbito público o desperdício financeiro também é verificado, no entanto, neste caso, o abandono significa recurso público investido sem o devido retorno, já que são alocados professores, funcionários, equipamentos e espaço físico cuja capacidade total não é integralmente desfrutada, devido à evasão (Silva Filho et al., 2007).

Considerando essas questões, Ambiel (2015) desenvolveu a Escala de Motivos para Evasão do Ensino Superior (M-ES), que se trata de um instrumento padronizado, no formato de um inventário, que avalia os motivos potenciais de estudantes ativos para deixarem seus respectivos cursos antes da conclusão. A M-ES permite identificar motivos relacionados à estrutura e ao funcionamento institucional, vivências pessoais e familiares, falta de suporte financeiro, carreira, desempenho acadêmico, relacionamentos interpessoais e autonomia envolvidos na evasão do curso superior.

É necessário que estudos desta natureza se façam presentes a fim de encontrar mais objetivamente os motivos que levam a evasão de um curso universitário. 
Neste sentido, o presente trabalho tem como objetivo verificar o poder preditivo dos fatores do QVA-r e do CAAS em relação aos motivos apontados pela Escala de Motivos para Evasão do Ensino Superior (M-ES) desenvolvida por Ambiel (2015). Considerando que o curso universitário demanda do estudante adaptações em sua forma de lidar com os estudos, com as relações interpessoais no contexto educacional e na forma de lidar com as aprendizagens e transições de carreira, a hipótese deste estudo é que o conjunto das variáveis independentes exerçam um maior poder preditivo sobre os motivos da evasão quando as vivencias acadêmicas e adaptabilidade de carreira forem insatisfatórias, exercendo algum poder preditivo para permanência no curso quando as vivencias acadêmicas e adaptabilidade de carreira forem satisfatórias.

\section{Método}

\section{Participantes}

Este estudo teve a participação de 153 acadêmicos de uma instituição privada de ensino superior do interior do estado de São Paulo. Os estudantes possuíam de 18 a 59 anos $(\mathrm{M}=25,6 ; \mathrm{DP}=8,59)$ sendo, em sua maioria, do sexo feminino $(n=114)$. Destes, $81 \%(n=124)$ eram estudantes de Psicologia e $17 \%(n=26)$ estudantes de Engenharia Elétrica. O semestre cursado variou de $2^{\circ}$ a $9^{\circ}(M=4,80 ; \mathrm{DP}=2,14)$. Vale destacar que a amostra foi por conveniência, o que explica a grande magnitude de idades.

\section{Instrumentos}

Questionário de Vivências Acadêmicas Versão Reduzida - QVA-r (Granado et al., 2005): A versão brasileira foi adaptada do instrumento elaborado em Portugal por Almeida, Soares e Ferreira (2002) para avaliar a qualidade das vivências acadêmicas do aluno em relação a si próprio, aos seus pares, aos professores e à instituição de ensino superior onde estuda. O questionário é respondido em uma escala likert que varia de "nada a ver comigo" (1 ponto) a "tudo a ver comigo" (5 pontos). A evidência de validade mais importante resultou da análise da estrutura interna dos itens, pela qual os 55 itens foram agrupados em seis dimensões, congruentes teoricamente: Carreira, Pessoal, Interpessoal, Estudo, Institucional e Compromisso com o curso. As estimativas de precisão são adequadas com valores de alfa de Cronbach acima de 0,70 .

Escala de Adaptabilidade de Carreira, versão brasileira (Career Adapt-Abilities Scale, CAASBrazil; Teixeira et al., 2012): A versão brasileira foi adaptada do CAAS-Portugal por Teixeira et al. (2012) com o propósito de avaliar as competências para lidar com tarefas de desenvolvimento de carreira. Os participantes responderam a cada um dos 22 itens, empregando uma escala likert de 1 a 5, indicando o quanto consideram que desenvolveram as competências descritas nos itens ao longo da vida. Pela análise da estrutura interna dos itens os autores identificaram uma estrutura de quatro fatores, tal como esperado, e, são denominados: consideração, controle, curiosidade e confiança. Considerando os resultados especificamente encontrados com a versão brasileira, os índices de precisão variaram entre 0,88 a 0,89 , e a escala total de obteve alfa de Cronbach $=0,91$.

Escala de Motivos para Evasão do Ensino Superior (M-ES; Ambiel, 2015): Trata-se de escala composta por 67 itens relacionados a motivos que influenciam na decisão de um aluno de graduação a evadir do seu curso superior. A chave de resposta é em formato likert de cinco pontos, variando entre "1 - muito fraco" e "5 - muito forte". O instrumento é subdividido em cinco fatores: Motivos Institucionais (Fator 1); Motivos Pessoais (Fator 2); Motivos relacionados à falta de suporte (Fator 3); Motivos relacionados à carreira (Fator 4); Motivos relacionados ao desempenho acadêmico (Fator 5); Motivos interpessoais (Fator 6) e Motivos relacionados à autonomia (Fator 7). Foram extraídos sete componentes principais com alfas de Cronbach entre 0,79 e 0,93 .

\section{Procedimentos}

Após aprovação do Comitê de Ética em Pesquisa da Universidade São Francisco (USF), a coleta de dados foi realizada, seguindo as exigências éticas. Os alunos foram convidados a participar espontaneamente da pesquisa durante um momento disponibilizado pelos professores em sala de aula, depois de prévia combinação com os coordenadores dos cursos. Após leitura e assinatura do Termo de Consentimento Livre e Esclarecido (TCLE) procedeu-se à aplicação do instrumento.

O preenchimento do questionário sociodemográfico, a ordem de aplicação dos demais instrumentos (CAAS, M-ES, QVA-r) seguiu de forma alternada para cada turma de alunos, buscando minimizar um possível efeito fadiga sobre os resultados dos testes. A aplicação dos testes ocorreu de forma coletiva em sala de aula, e com duração média de 40 minutos.

\section{Análise de dados}

Os resultados foram analisados com o software Statistical Package for the Social Sciences (SPSS), versão 20. Além da estatística descritiva, recorrendo- 
se à análise de correlação de Pearson, cujas magnitudes foram interpretadas com base em Dancey e Reidy (2013) que consideram que valores de $r$ na casa de 0 são nulas; entre 0,10 e 0,39 são fracos; ente 0,40 e 0,69 são moderados; entre 0,70 e 0,90 são fortes; e valores acima de 0,90 representam correlações perfeitas. Foi também empregada a regressão linear múltipla, buscando compreender as relações entre as variáveis testadas na Escala de Motivos para Evasão do Ensino Superior (variáveis dependentes) em relação aos dados coletados no Questionário de Vivência Acadêmica Reduzido e na Escala de Adaptabilidade de Carreira (variáveis independentes).

\section{Resultados}

Inicialmente, foi analisada a correlação entre os instrumentos. A Tabela 1 mostra os resultados obtidos entre a M-ES e o QVA-r.

TABELA 1

Correlações entre as dimensões M-ES e do QVA-r.

\begin{tabular}{|c|c|c|c|c|c|}
\hline & $\begin{array}{c}\text { QVA-r } \\
\text { Carreira }\end{array}$ & $\begin{array}{c}\text { QVA-r } \\
\text { Pessoal }\end{array}$ & $\begin{array}{c}\text { QVA-r } \\
\text { Interpessoal }\end{array}$ & $\begin{array}{l}\text { QVA-r } \\
\text { Estudo }\end{array}$ & $\begin{array}{c}Q V A-r \\
\text { Institucional }\end{array}$ \\
\hline $\begin{array}{l}M \text {-ES } \\
\text { Carreira }\end{array}$ & $-0,27 *$ & 0,15 & $-0,01$ & 0,01 & $-0,10$ \\
\hline $\begin{array}{l}\text { M-ES } \\
\text { Desempenho } \\
\text { Acadêmico }\end{array}$ & $-0,24 *$ & $0,25^{*}$ & $-0,03$ & $0,23 *$ & $-0,05$ \\
\hline $\begin{array}{l}\text { M-ES } \\
\text { Interpessoais }\end{array}$ & $-0,29^{*}$ & $0,26^{*}$ & $-0,11$ & 0,08 & $-0,11$ \\
\hline $\begin{array}{l}\text { M-ES } \\
\text { Autonomia }\end{array}$ & $-0,17$ & $0,27^{*}$ & $-0,15$ & 0,15 & $-0,11$ \\
\hline $\begin{array}{l}\text { M-ES } \\
\text { Institucionais }\end{array}$ & $-0,27 * *$ & ,098 & $-0,04$ & 0,09 & $-0,06$ \\
\hline $\begin{array}{l}\text { M-ES } \\
\text { Vocacionais e } \\
\text { Pessoais }\end{array}$ & $-0,14$ & 0,07 & $-0,05$ & 0,01 & $-0,05$ \\
\hline $\begin{array}{l}\text { M-ES } \\
\text { Falta de } \\
\text { Suporte }\end{array}$ & $-0,19$ & 0,01 & $-0,02$ & 0,03 & $-0,044$ \\
\hline
\end{tabular}

$* \mathrm{p}<0,05 ; * * \mathrm{p}<0,01$.

O fator do QVA-r, Carreira, teve correlação com quatro das dimensões da M-ES. Os índices foram negativos e fracos $(0,24$ a 0,27$)$. Por sua vez, o QVA-r Pessoal correlacionou-se com três das dimensões da M-ES, sendo os índices positivos e fracos $(0,25$ a 0,27$)$. Por fim, o QVA-r Estudo apresentou uma correlação fraca e positiva com a dimensão Desempenho Acadêmico da M-ES. A seguir, na Tabela 2 serão apresentados os resultados das correlações realizadas entre M-ES e CAAS.
TABELA 2

Correlações entre as dimensões M-ES e da CAAS.

\begin{tabular}{|c|c|c|c|c|}
\hline & $\begin{array}{c}C A A S \\
\text { Consideração }\end{array}$ & $\begin{array}{c}C A A S \\
\text { Controle }\end{array}$ & $\begin{array}{c}C A A S \\
\text { Curiosidade }\end{array}$ & $\begin{array}{c}C A A S \\
\text { Confiança }\end{array}$ \\
\hline $\begin{array}{l}M \text {-ES } \\
\text { Carreira }\end{array}$ & $-0,19 *$ & $-0,12$ & $-0,13$ & $-0,10$ \\
\hline $\begin{array}{l}\text { M-ES } \\
\text { Desempenho } \\
\text { Acadêmico }\end{array}$ & $-0,10$ & $-0,12$ & $-0,06$ & $-0,16^{*}$ \\
\hline $\begin{array}{l}\text { M-ES } \\
\text { Interpessoais }\end{array}$ & $-0,21 * *$ & $-0,15$ & $-0,06$ & $-0,18 *$ \\
\hline $\begin{array}{l}\text { M-ES } \\
\text { Autonomia }\end{array}$ & $-0,26 * *$ & $-0,32 * *$ & $-0,13$ & $-0,12$ \\
\hline $\begin{array}{l}\text { M-ES } \\
\text { Institucionais }\end{array}$ & $-0,12$ & 0,08 & 0,10 & 0,08 \\
\hline $\begin{array}{l}\text { M-ES } \\
\text { Vocacionais e } \\
\text { Pessoais }\end{array}$ & $-0,08$ & $-0,02$ & $-0,04$ & 0,07 \\
\hline $\begin{array}{l}\text { M-ES } \\
\text { Falta de } \\
\text { Suporte }\end{array}$ & $-0,15$ & $-0,04$ & 0,03 & $-0,07$ \\
\hline
\end{tabular}

Dentre os resultados encontrados, evidencia-se que todas as correlações significativas encontradas foram negativas e fracas. $O$ fator Consideração da CAAS correlacionou-se com três fatores da M-ES $(0,19$ a 0,26$)$, enquanto que o fator Confiança da CAAS correlacionou com dois fatores de motivos para evasão $(0,16$ a 0,18$)$. $O$ fator Controle correlacionou-se apenas com motivos relacionados à Autonomia, com o maior coeficiente observado. Nas Tabelas 3, 4 e 5 estão apresentados os resultados da análise de regressão linear múltipla com os fatores da M-ES que tiveram coeficientes significativos com QVA-r e CAAS.

No primeiro modelo foram inseridos apenas os fatores do QVA-r, que, juntos, explicaram 11,3\% da variação a do fator Motivos relacionados à Carreira da M-ES. No segundo modelo, foram inseridos também os fatores da CAAS e, nesse caso, houve um acréscimo na porcentagem de variação explicada, sendo que todas as variáveis (QVA-r e CAAS) em conjunto explicaram $18 \%$. De forma específica, observa-se que o fator Consideração da CAAS foi um preditor significativo dos Motivos de evasão relacionados à Carreira, sendo que a cada ponto a mais em Consideração é esperada a diminuição de aproximadamente um quarto de desvio-padrão em tais motivos. Além disso, apesar de não terem sido preditores significativos, os fatores Carreira e Pessoal do QVA-r e Confiança, da CAAS, também apresentaram coeficientes beta padronizado consideráveis. Na Tabela 4, podem ser observados os resultados referentes ao fator Interpessoal da M-ES. 
TABELA 3

Análise de Regressão Linear Múltipla do M-ES - Carreira em relação aos construtos do QVA-r e CAAS.

\begin{tabular}{|c|c|c|c|c|}
\hline & Modelo & $\begin{array}{c}\text { Coef. Padronizado } \\
\text { Beta }\end{array}$ & $t$ & Sig. \\
\hline \multirow{7}{*}{ Modelo 1} & Constante & & 2,996 & 0,004 \\
\hline & QVA-r Carreira & $-0,299$ & $-2,531$ & 0,013 \\
\hline & QVA-r Pessoal & 0,151 & 1,364 & 0,176 \\
\hline & QVA-r Interpessoal & 0,127 & 1,078 & 0,284 \\
\hline & QVA-r Estudo & 0,076 & 0,667 & 0,507 \\
\hline & QVA-r Institucional & $-0,033$ & $-0,251$ & 0,803 \\
\hline & $\mathrm{R}^{2}$ & \multicolumn{2}{|c|}{$11,3 \%$} & \\
\hline \multirow{11}{*}{ Modelo 2} & Constante & & 2,022 & 0,047 \\
\hline & QVA-r Carreira & $-0,223$ & $-1,805$ & 0,075 \\
\hline & QVA-r Pessoal & 0,195 & 1,740 & 0,086 \\
\hline & QVA-r Interpessoal & 0,144 & 1,189 & 0,238 \\
\hline & QVA-r Estudo & 0,093 & 0,789 & 0,433 \\
\hline & QVA-r Institucional & $-0,086$ & $-0,641$ & 0,523 \\
\hline & CAAS Consideração & $-0,263$ & $-2,045$ & 0,044 \\
\hline & CAAS Controle & 0,137 & 0,983 & 0,329 \\
\hline & CAAS Curiosidade & $-0,114$ & $-0,812$ & 0,419 \\
\hline & CAAS Confiança & 0,217 & 1,572 & 0,120 \\
\hline & $\mathrm{R}^{2}$ & \multicolumn{2}{|c|}{$18 \%$} & \\
\hline
\end{tabular}

TABELA 4

Análise de Regressão Linear Múltipla do M-ES Interpessoal em relação aos construtos do QVA-r e CAAS.

\begin{tabular}{|c|c|c|c|c|}
\hline & Modelo & $\begin{array}{c}\text { Coef. Padronizado } \\
\text { Beta }\end{array}$ & $t$ & Sig. \\
\hline \multirow{7}{*}{ Modelo 1} & Constante & & 3,105 & 0,003 \\
\hline & QVA-r Carreira & $-0,238$ & $-2,023$ & 0,046 \\
\hline & QVA-r Pessoal & 0,237 & 2,148 & 0,035 \\
\hline & QVA-r Interpessoal & 0,028 & 0,237 & 0,814 \\
\hline & QVA-r Estudo & 0,035 & 0,305 & 0,761 \\
\hline & QVA-r Institucional & $-0,034$ & $-0,257$ & 0,798 \\
\hline & $\mathrm{R}^{2}$ & \multicolumn{2}{|c|}{$12 \%$} & \\
\hline \multirow{11}{*}{ Modelo 2} & Constante & & 3,377 & 0,001 \\
\hline & QVA-r Carreira & $-0,173$ & $-1,403$ & 0,165 \\
\hline & QVA-r Pessoal & $-0,246$ & 2,197 & 0,031 \\
\hline & QVA-r Interpessoal & 0,069 & 0,567 & 0,573 \\
\hline & QVA-r Estudo & 0,106 & 0,897 & 0,373 \\
\hline & QVA-r Institucional & $-0,062$ & $-0,464$ & 0,644 \\
\hline & CAAS Consideração & $-0,216$ & $-1,675$ & 0,098 \\
\hline & CAAS Controle & $-0,045$ & $-0,326$ & 0,745 \\
\hline & CAAS Curiosidade & 0,143 & 1,014 & 0,314 \\
\hline & CAAS Confiança & $-0,114$ & $-0,825$ & 0,412 \\
\hline & $\mathrm{R}^{2}$ & \multicolumn{2}{|c|}{$17,8 \%$} & \\
\hline
\end{tabular}


TABELA 5

Análise de Regressão Linear Múltipla do M-ES Autonomia em relação aos construtos do QVA-r e CAAS.

\begin{tabular}{|c|c|c|c|c|}
\hline & Modelo & $\begin{array}{c}\text { Coef. Padronizado } \\
\text { Beta }\end{array}$ & $t$ & Sig. \\
\hline \multirow{7}{*}{ Modelo 1} & Constante & & 3,067 & 0,003 \\
\hline & QVA-r Carreira & $-0,066$ & $-0,547$ & 0,586 \\
\hline & QVA-r Pessoal & 0,202 & 1,794 & 0,076 \\
\hline & QVA-r Interpessoal & $-0,092$ & $-0,769$ & 0,444 \\
\hline & QVA-r Estudo & $-0,051$ & $-0,443$ & 0,659 \\
\hline & QVA-r Institucional & $-0,057$ & $-0,426$ & 0,671 \\
\hline & $\mathrm{R}^{2}$ & \multicolumn{2}{|c|}{$8 \%$} & \\
\hline \multirow{11}{*}{ Modelo 2} & Constante & & 3,574 & 0,001 \\
\hline & QVA-r Carreira & $-0,024$ & $-0,194$ & 0,847 \\
\hline & QVA-r Pessoal & 0,215 & 1,945 & 0,055 \\
\hline & QVA-r Interpessoal & $-0,038$ & $-0,318$ & 0,751 \\
\hline & QVA-r Estudo & 0,016 & 0,135 & 0,893 \\
\hline & QVA-r Institucional & $-0,077$ & $-0,580$ & 0,563 \\
\hline & CAAS Consideração & $-0,226$ & $-1,776$ & 0,080 \\
\hline & CAAS Controle & $-0,291$ & $-2,121$ & 0,037 \\
\hline & CAAS Curiosidade & 0,054 & 0,390 & 0,698 \\
\hline & CAAS Confiança & 0,209 & 1,538 & 0,128 \\
\hline & $\mathrm{R}^{2}$ & \multicolumn{2}{|c|}{$19 \%$} & \\
\hline
\end{tabular}

Os resultados da Tabela 4 mostram que a inclusão dos fatores da CAAS no modelo explicativo aumentou de forma importante a porcentagem de variância explicada em relação aos motivos interpessoais para evasão. De forma específica, nota-se que o fator Pessoal do QVA-r foi o único preditor significativo, com um coeficiente beta padronizado positivo de 0,25 . A Tabela 5 evidencia os resultados com o fator Autonomia.

Nesse caso, observa-se que as variáveis da CAAS foram as que mais contribuíram para a explicação dos motivos, sendo que sua inclusão no modelo implicou no dobro de variância explicada que anteriormente era apenas explicada pelos fatores do QVA-r. Especificamente, o fator Controle da CAAS foi um preditor significativo, com um coeficiente próximo a -0,30.

\section{Discussão}

Este estudo teve como objetivo avaliar as relações entre as vivências acadêmicas, a adaptabilidade de carreira e os motivos para evasão no ensino superior. De forma específica, procurou-se avaliar o poder preditivo das vivências e da adaptabilidade de carreira em relação aos motivos para evasão.

Dentre os resultados, cabe destacar que diversas correlações significativas, ainda que em geral fracas, foram encontradas tanto em relação às vivências acadêmicas quanto à adaptabilidade de carreira em relação aos motivos para evasão. Especialmente as variáveis dos instrumentos relacionadas à carreira mostraram-se mais relacionadas, de forma negativa, com os motivos para evasão, o que pode levar a pensar que as expectativas que os estudantes têm acerca de sua carreira no futuro podem estar bastante relacionadas com as decisões de permanência ou desistência do curso. Estes achados vão ao encontro de achados anteriores descritos na literatura (Almeida et al., 2003; Astin, 1993; Chickering \& Reisser, 1993; Granado et al., 2005; Tinto, 1993).

Entre as dimensões da escala de motivos de evasão do ensino superior (M-ES) e os indicadores da escala de adaptabilidade de carreira (CAAS), observou-se correlações negativas e fracas com todos os domínios da CAAS. Chama a atenção a correlação encontrada entre Controle (CAAS) e Autonomia (M-ES), por conta do maior coeficiente encontrado, ainda que baixo. Esse resultado aponta que pessoas que se responsabilizam pelas próprias decisões de carreira tendem a ter menos motivos relacionados à autonomia para um bom desenvolvimento no curso (Ambiel, 2015; Teixeira et al., 2012). 
O objetivo principal do estudo era testar o poder preditivo da adaptação acadêmica e da adaptabilidade de carreira em relação aos motivos para evasão. Para tanto, foram realizadas análises de regressão individualmente para cada fator da M-ES, sempre testando dois modelos: o primeiro, contendo apenas os fatores do QVA-r como variáveis independentes e, o segundo, incluindo também os fatores da CAAS. De forma geral, observou-se que a inclusão dos fatores da CAAS no modelo da regressão aumentou consideravelmente o total explicado dos motivos para evasão para três das sete dimensões da M-ES, quais sejam, motivos relacionados à carreira, motivos interpessoais e de autonomia. Esse resultado sugere que o desenvolvimento de habilidades de adaptação de carreira pode exercer um papel favorável sobre as intenções de continuidade no curso de formação atual e progressão futura para a inclusão no mercado de trabalho, o que encontra respaldo nos achados prévios de Duffy et al. (2015) e Monteiro e Almeida (2015).

Nos resultados encontrados, o fator Consideração da CAAS mostrou-se como preditor negativo dos motivos para evasão relacionados à carreira. O referido fator de adaptabilidade diz respeito às atitudes de planejar e refletir sobre sua situação atual e futura na carreira escolhida (Savickas \& Porfeli, 2012). Tendo isso em vista, é interessante que o aumento dessa habilidade tenha um efeito na diminuição dos motivos potenciais para deixar o curso por conta de decepção com a profissão escolhida (Ambiel, 2015). Esse resultado reforça a importância de comportamentos exploratórios, de si e do ambiente, por parte do aluno que cursa o ensino superior (Astin, 1993; Chickering \& Reisser, 1993; Silva et al., 2013).

Outro fator da CAAS que se mostrou um preditor significativo de motivos para evasão foi Controle, em relação aos motivos relacionados à autonomia. Este resultado informa que a competência para se responsabilizar pelas próprias decisões de carreira (Savickas \& Porfeli, 2012), característica própria do fator Controle da CAAS, implica na diminuição da força dos motivos relacionados à dependência de outros para se manter no curso em relação à moradia e traslado até a instituição, que são motivos para evasão relacionados à autonomia (Ambiel, 2015).

Além desses, vale comentar também que o aumento dos motivos para evasão relacionados às relações interpessoais pouco satisfatórias com colegas, professores e funcionários da instituição de ensino (Ambiel, 2015) é predito significativamente pela diminuição da pontuação no fator Pessoal do QVA-r, que diz respeito ao bem-estar geral sentido no ambiente acadêmico (Almeida et al., 1999; Granado et al., 2005). Em outras palavras, o resultado revela que as pessoas que se sentem bem e confortáveis emocionalmente tendem a perceber como mais fracos os motivos para a evasão por conta de maus relacionamento neste contexto, dados que encontram respaldo nos resultados de Bardagi e Hutz (2005) e Tinto (1975, 1993). Esses autores ressaltam que, para além da estrutura física da instituição, os relacionamentos estabelecidos, quando saudáveis e positivos, podem contribuir para a permanência e conclusão do curso.

Os resultados encontrados sugerem, portanto, que o investimento das instituições de ensino superior na manutenção dos alunos nos cursos passa por providenciar boas condições de relacionamento e bemestar geral, além da disponibilização de possibilidades para o desenvolvimento de habilidades para lidar com as decisões de carreira. Esses dados vão ao encontro do que Tinto $(1975,2007)$ preconizava como sendo prerrogativa de instituições que prezam por conduzir seus estudantes até a formatura. $\mathrm{O}$ autor destaca ainda que, para tanto, não seriam necessários grandes investimentos adicionais em programas complexos de retenção, mas em pequenas ações cotidianas que podem aumentar a sensação de bem-estar e pertencimento do aluno à instituição. Além disso, Tinto (2007) destaca o importante papel que o professor tem em sala de aula, no sentido tanto de promover um ambiente acolhedor quanto ao ser um modelo em relação à carreira, devendo, oportunamente, integrar a discussão sobre a escolha de carreira ao conteúdo programático das disciplinas teóricas e práticas. Como afirmam Teixeira e Gomes (2005) seria importante que durante o ensino superior houvesse maior ênfase nas práticas profissionais e na instrumentalização dos estudantes para a transição para o mercado de trabalho, possibilitando não apenas a reflexão, mas também a responsabilização sobre a sua trajetória acadêmica e profissional.

Do ponto de vista teórico, este estudo contribui ao identificar que, ao se considerar o estudo dos motivos individuais para a evasão, as questões de adaptação acadêmica não devem ser observadas de forma independente àquelas relacionadas à carreira, ou seja, os resultados deste estudo sugerem que a adaptação de forma ampla, tanto relacionada ao ensino superior quanto à carreira, precisam ser consideradas para lidar com a evasão. Importa notar que, ao menos no Brasil, estudos anteriores limitaram-se a identificar as estatísticas sobre a evasão em amostras de larga escala (Lobo, 2012; Silva Filho et al., 2007) sem considerar os motivos que conduziram a tal decisão, ou relacionando-a apenas a questões vocacionais (relativas à escolha profissional inicial) ou financeiras (Bardagi 
\& Hutz, 2005). Assim, os resultados do presente estudo parecem sugerir que os aspectos relacionados à carreira devem ser mais fortemente abrangidos pelos estudos sobre adaptação acadêmica.

À guisa de conclusão pode-se afirmar que os objetivos deste trabalho foram atingidos, com base nos seus resultados sugere-se que no futuro seja posta à prova a premissa de que o envolvimento com a carreira durante o curso de graduação exerce influência na decisão de manter-se ou de deixar o curso. Para tanto, sugere-se a realização de estudos longitudinais e que avaliem outras variáveis relacionadas à vivência acadêmica e à evasão de cursos e instituições de ensino, tais como expectativas quanto a carreira, satisfação com o curso, personalidade, desempenho acadêmicos, entre outros.

Ademais, algumas limitações devem ser delineadas. A principal diz respeito à amostra, advinda de uma única instituição e pouco diversificada em termos da proveniência dos alunos. Como foram investigados apenas dois cursos, com predominância de estudantes de Psicologia e mulheres, é possível que o enfoque nos aspectos relacionais, aparente nos resultados, tenha se dado, ao menos em parte, por conta de características próprias dos estudantes desse curso. Além disso, é possível que o ambiente da instituição na qual a coleta foi feita também tenha tido alguma influência nos resultados, fato pelo qual a diversidade de experiências de relacionamento entre o estudante e a instituição deve ser privilegiada no futuro.

Ainda assim, são dados que ajudam a vislumbrar que as universidades necessitam estar mais bem preparadas para assumir o tema da evasão como um fenômeno que pode ser prevenido e que depende de uma estrutura psicopedagógica a qual pode ser oferecida pelas instituições aos seus alunos. Muitos estudos indicam este mesmo caminho e entende-se que a implementação de estratégias pelas instituições de ensino pode minimizar o impacto das dificuldades vivenciadas pelos alunos por ocasião do ingresso na universidade. A intervenção da instituição sobre os fatores de risco para a evasão tende a facilitar a adaptação acadêmica e, consequentemente, um maior investimento do aluno na sua carreira. Uma implicação prática a ser destacada diz respeito à necessidade de que as IES, considerando a diversidade dos cursos oferecidos, deem maior atenção à disseminação de informações e ao oferecimento de espaços para reflexão e discussão sobre a carreira.

\section{Referências}

Almeida, L. S., Ferreira, J. A. G., \& Soares, A. P. C. (1999). Questionário de Vivências Académicas: Construção e validação de uma versão reduzida (QVA-r). Revista Portuguesa de Pedagogia, 3, 181-207.

Almeida, L. S., Ferreira, J. A., \& Soares, A. P. (2003). Questionário de Vivências Académicas (QVA e QVA-r). In M. M. Gonçalves, M. R. Simões, L. S. Almeida, \& C. Machado (Orgs.), Avaliação psicológica: Instrumentos validados para a população portuguesa (pp. 113-130). Coimbra: Quarteto Editora.

Almeida, L. S., Soares, A. P., \& Ferreira, J. A. G. (2002). Questionário de Vivências Acadêmicas (QVA-r): Avaliação do ajustamento dos estudantes universitários. Avaliação Psicológica, 2, 81-93.

Ambiel, R. A. (2015). Construção da Escala de Motivos para Evasão do Ensino Superior. Avaliaçao Psicologica: Interamerican Journal of Psychological Assessment, 14(1), 41-52. http://dx.doi.org/10.15689/ap.2015.1401.05

Araújo, A. M., Almeida, L. S., Costa, A. R., Alfonso, S., Conde, Á., \& Dea-o, M. (2015). Variáveis pessoais e socioculturais de diferenciação das expectativas académicas: Estudo com alunos do Ensino Superior do Norte de Portugal e Galiza. Revista Portuguesa de Educação, 28(1), 201-220. http://dx.doi.org/10.21814/rpe.7057

Astin, A. (1993). What matters in college? Four critical years revised. San Francisco, US: Jossey-Bass. http://dx.doi. org/10.2307/1176821

Bardagi, M. P., \& Hutz, C. S. (2005). Evasão e serviços de apoio ao estudante: Uma breve revisão da literatura brasileira. Psicologia Revista, 14(2), 279-301.

Bardagi, M. \& Hutz, C. S. (2014). Evasão universitária e serviços de apoio ao estudante: uma breve revisão da literatura brasileira. Psicologia Revista - Revista da Faculdade de Ciências Humanas e da Saúde, 14(2), 279-301.

Brasil. Ministério da Educação e Cultura. Secretaria de Ensino Superior. (1996). Comissão Especial de Estudos sobre a Evasão nas Universidades Públicas Brasileiras. Brasília, DF: MEC/SESU.

Brasil. Presidência da República. (2005). Lei no 11.096. Institui o programa Universidade para todos- PROUNI. Brasília, DF: Casa Civil.

Brasil. Presidência da República. (2007). Decreto no 6.096. Institui o Programa de Apoio a Planos de Reestruturação e Expansão das Universidades Federais - REUNI. Brasília, DF: Casa Civil.

Cabrera, L., Bethencourt, J. T., Pérez, P. A., \& Afonso, M. G. (2006). El problema del abandono de los estudios universitarios. Revista Electrónica de Investigación y Evaluación Educativa, 12(2), 171-203.

Chickering, A. W. \& Reisser, L. (1993). Education and identity. San Francisco, US: Jossey-Bass. 
Dancey, C. P. \& Reidy, J. (2013). Estatística sem matemática para psicologia: usando o SPSS para Windows. Porto Alegre: Artmed.

Duffy, R. D., Douglass, R. P., \& Autin, K. L. (2015). Career adaptability and academic satisfaction: Examining work volition and self-efficacy as mediators. Journal of Vocational Behavior, 90, 46-54. http://dx.doi.org/10.1016/j. jvb.2015.07.007

Granado, J. I. F., Santos, A. A. A., Almeida, L. S., Soares, A. P., \& Guisande, M. A. (2005). Integração académica de estudantes universitários: Contributos para a adaptação e validação do QVA-r no Brasil. Psicologia e Educação, 4(2), 31-41.

Igue, E. A., Bariani, I. C. D., \& Milanesi, P. V. B. (2008). Vivência acadêmica e expectativas de universitários ingressantes e concluintes. Psico-USF, 13(2), 155-164.

Í-iguez, T, Saso, C. E., \& Errazu, D. V. (2016). La Universidad del Espacio Europeo de Educación Superior ante el abandono de los estudios de grado. Causas y propuestas estratégicas de prevención. Educar, 52(2), 285-313. http://dx.doi.org/10.5565/rev/educar.674

Lobo, M. B. D. C. M. (2012). Panorama da evasão no ensino superior brasileiro: aspectos gerais das causas e soluções. Cadernos, 25, 1-23.

Monteiro, S. \& Almeida, L. S. (2015). The relation of career adaptability to work experience, extracurricular activities, and work transition in Portuguese graduate students. Journal of Vocational Behavior, 91, 106-112. http://dx.doi.org/10.1016/j.jvb.2015.09.006

Noronha, A. P. P., Martins, D. F., Gurgel M. G. A., \& Ambiel, R. A. M. (2009). Estudo correlacional entre interesses profissionais e vivências acadêmicas no ensino superior. Revista Semestral da Associação Brasileira de Psicologia Escolar e Educacional (ABRAPEE), 13(1), 143-154.

Oliveira, R. E. C. \& Morais, A. (2015). Vivências acadêmicas e adaptação de estudantes de uma universidade pública federal do Estado do Paraná. Revista de Educação Pública, 24(57), 547-568.

Savickas, M. L. (1997). Career adaptability: An integrative construct for life-span, life-space theory. Career Development Quarterly, 45, 247-259. http://dx.doi.org/10.1002/j.2161-0045.1997.tb00469.x

Savickas, M. L. (2005). The theory and practice of career construction. In S. D. Brown \& R. W. Lent (Eds.), Career development and counselling: Putting theory and research to work (pp. 42-70). Hoboken, NJ: Wiley.

Savickas, M. L. \& Porfeli, E. J. (2012). Career Adapt-Abilities Scale: Construction, reliability, and measurement equivalence across 13 countries. Journal of Vocational Behavior, 80(3), 661-673. http://dx.doi.org/10.1016/j. jvb.2012.01.011

Silva Filho, R. L. L., Motejunas, P. R., Hipólito, O., \& Lobo, M. B. C. M. (2007). A evasão no ensino superior brasileiro. Cadernos de Pesquisa, 37(132), 641-659. http://dx.doi.org/10.1590/S0100-15742007000300007

Silva, C. S. C. D., Coelho, P. B. M., \& Teixeira, M. A. P. (2013). Relações entre experiências de estágio e indicadores de desenvolvimento de carreira em universitários. Revista Brasileira de Orientação Profissional, 14(1), 35-46.

Sousa, H. D., Bardagi, M. P., \& Nunes, C. H. S. D. S. (2013). Autoeficácia na formação superior e vivências de universitários cotistas e não cotistas. Avaliação Psicológica, 12(2), 253-261.

Teixeira, M. A. P., \& Gomes, W. B. (2005). Decisão de carreira entre estudantes em fim de curso universitário. Psicologia: Teoria e Pesquisa, 21(3), 327-334. http://dx.doi.org/10.1590/S0102-37722005000300009

Teixeira, M. A. P., Bardagi, M. P., Lassance, M. C. P., Magalhães, M. O., \& Duarte, M. E. (2012). Career adapt-abilities scale - Brazilian form: psychometric properties and relationships to personality. Journal of Vocational Behavior, $80(3), 680-685$.

Teixeira, M. A. P., Castro, G. D., \& Piccolo, L. R. (2007). Adaptação à universidade em estudantes universitários: um estudo correlacional. Interação em Psicologia, 11(2), 211-220. http://dx.doi.org/10.5380/psi.v11i2.7466

Tinto, V. (1975). Dropout from higher education: A theoretical synthesis of recent research. Review of Educational Research, 45(1), 89-125. http://dx.doi.org/10.3102/00346543045001089

Tinto, V. (1993). Leaving college: Rethinking the causes and cures of student attrition (2 ${ }^{\mathrm{a}}$ ed.). Chicago, US: University of Chicago Press.

Tinto, V. (2007). Research and practice of student retention: what next? Journal of College Student Retention, 8(1), 1-19. http://dx.doi.org/10.2190/4YNU-4TMB-22DJ-AN4W

\footnotetext{
Autores:

Endereço para correspondência:

Rodolfo A. M. Ambiel

Rua Alexandre Rodrigues Barbosa, 45 - Centro

13251-900 Itatiba, SP, Brasil

<rodolfo.ambiel@usf.edu.br>

Recebido em: 01.05.2016

Aceito em: 23.08.2016
}

Rodolfo Augusto Matteo Ambiel - Doutor, Universidade São Francisco.

Acácia Aparecida Angeli dos Santos - Doutora, Universidade São Francisco.

Simone Nenê Portela Dalbosco - Doutoranda, Faculdade Meridional. 